\title{
LEBESGUE POINTS AND CAPACITIES VIA BOXING INEQUALITY IN METRIC SPACES
}

\author{
JUHA KINNUNEN, RIIKKA KORTE, NAGESWARI SHANMUGALINGAM \\ AND HELI TUOMINEN
}

\begin{abstract}
The purpose of this work is to study regularity of Sobolev functions on metric measure spaces equipped with a doubling measure and supporting a weak Poincaré inequality. We show that every Sobolev function whose gradient is integrable to power one has Lebesgue points outside a set of 1-capacity zero. We also show that 1-capacity is equivalent to the Hausdorff content of codimension one and study characterizations of 1-capacity in terms of Frostman's lemma and functions of bounded variation. As the main technical tool, we prove a metric space version of Gustin's boxing inequality. Our proofs are based on covering arguments and functions of bounded variation. Perimeter measures, isoperimetric inequalities and coarea formula play an essential role in our approach.
\end{abstract}

\section{INTRODUCTION}

We study first order Sobolev spaces in the metric space setting. Sobolev spaces are classically defined as $p$ th power integrable functions whose weak gradient is integrable to power $p$ with $1 \leq p<\infty$. In metric measure spaces, there are several alternative definitions available, but in general these definitions do not give the same class of functions when $p=1$. We use a definition based on upper gradients which gives the standard Sobolev space in the Euclidean case with Lebesgue measure also for $p=1$, see [Sh]. We use the rather standard assumptions that the measure is doubling and the space supports a weak Poincaré inequality.

Exceptional sets for Sobolev functions are measured in terms of the $p$-capacity. The theory is relatively complete in the case $1<p<$ $\infty$, but when $p=1$, somewhat unexpected phenomena occur even in the Euclidean case with Lebesgue measure. Indeed, when $1<p<$

2000 Mathematics Subject Classification. 46E35.

The research is supported by the NSF grant DMS-0355027 and the Finnish Academy of Science and Letters, the Vilho, Yrjö and Kalle Väisälä Foundation. Part of the research was done while the authors visited the University of Cincinnati in 2005-2006. We would like to thank the Taft Foundation for generous support. 
$\infty$, the $p$-capacity and the Hausdorff content of codimension $p$ are not equivalent, see the discussion, for example, in [FZ] and [EG]. In practice, this means that when $1<p<\infty$, we always seem to lose information if we pass from capacitary estimates to Hausdorff type estimates (or vice versa). However, as the 1-capacity is equivalent by two sided estimates to the Hausdorff content of codimension one, measure theoretic arguments are available. In the Euclidean setting this has been obtained in [Fl], see also [Ma2] and [MSZ]. Analogous results have been studied in the setting of weighted Euclidean case in $[\mathrm{Tu}]$ and in the metric space setting by [Ma1]. Indeed, our work is closely related to the paper [Ma1] of Malý, where similar results have been obtained by a different method. One of our goals is to further study this phenomenon in metric measure spaces. As our results apply to more general sets than level sets of Sobolev functions, some readers may find our approach interesting even in the Euclidean case with Lebesgue measure.

Our main tool is the boxing inequality, which is originally due to Gustin $[\mathrm{Gu}]$. In the Euclidean case, the boxing inequality states that every compact set $K \subset \mathbb{R}^{n}$ can be covered by balls $B\left(x_{i}, r_{i}\right), i=$ $1,2, \ldots$, in such a way that

$$
\sum_{i=1}^{\infty} r_{i}^{n-1} \leq c H^{n-1}(\partial K),
$$

where the constant $c$ depends only on the dimension $n$. Here $H^{n-1}$ refers to the $(n-1)$-dimensional Hausdorff measure. We give a proof of a metric space version of this result, which may be of independent interest. Our proof is based on a Calderón-Zygmund type decomposition and functions of bounded variation studied by Miranda [Mi]. Perimeter measures, isoperimetric inequalities and coarea formula play an essential role in our approach.

We give two applications of the boxing inequality. As the first application, we show equivalence of the capacity and Hausdorff content when $p=1$, and then we prove that Sobolev functions have Lebesgue points except on a set of capacity zero. This result is based on a capacitary weak type estimate for the Hardy-Littlewood maximal function. This estimate is usually proved by using the Besicovitch covering theorem, extension results or representation formulas for Sobolev functions, see [FZ] and [EG]. We do not have these tools available. When $1<p<\infty$, we may also use maximal function arguments (see [KL]), but this approach fails as well when $p=1$. Instead we apply the boxing inequality, coarea formula and covering arguments in our proof.

We also study several characterizations of the 1-capacity in the spirit of [FZ]. In particular, we consider connections to Frostman's lemma and 
functions of bounded variation. In the final section, we study conditions called $p$-hyperbolicity and $p$-parabolicity introduced by Holopainen in [Ho]. These conditions are related to non-triviality of the variational capacity.

\section{Preliminaries}

We assume that $X=(X, d, \mu)$ is a complete metric measure space equipped with a metric $d$ and a Borel regular outer measure $\mu$ such that $0<\mu(B)<\infty$ for all balls $B=B(x, r)=\{y \in X: d(x, y)<r\}$. For $\tau>0$, we write $\tau B=B(x, \tau r)$.

The measure $\mu$ is said to be doubling if there exists a constant $c_{D} \geq 1$, called the doubling constant of $\mu$, such that

$$
\mu(2 B) \leq c_{D} \mu(B)
$$

for all balls $B$ of $X$.

In this paper, a path in $X$ is a rectifiable nonconstant continuous mapping from a compact interval to $X$. A path can thus be parameterized by arc length.

We define Sobolev spaces on $X$ using upper gradients, see Shanmugalingam $[\mathrm{Sh}]$.

Definition 2.1. A nonnegative Borel function $g$ on $X$ is an upper gradient of an extended real valued function $u$ on $X$ if for all paths $\gamma$ joining points $x$ and $y$ in $X$ we have

$$
|u(x)-u(y)| \leq \int_{\gamma} g d s,
$$

whenever both $u(x)$ and $u(y)$ are finite, and $\int_{\gamma} g d s=\infty$ otherwise. If $g$ is a nonnegative measurable function on $X$ and if (2.2) holds for $p$-almost every path, then $g$ is a $p$-weak upper gradient of $u$.

By saying that (2.2) holds for $p$-almost every path with $1 \leq p<\infty$, we mean that it fails only for a path family with zero $p$-modulus. A family $\Gamma$ of curves is of zero $p$-modulus if there is a non-negative Borel measurable function $\rho \in L^{p}(X)$ such that for all curves $\gamma \in \Gamma$, the path integral $\int_{\gamma} \rho d s$ is infinite. Note that if $g$ is a $p$-weak upper gradient of $u$ and $\rho$ is such a function, then $g+\varepsilon \rho$ is an upper gradient of $u$ for all $\varepsilon>0$.

Definition 2.3. Let $1 \leq p<\infty$. If $u$ is a function that is integrable to power $p$ in $X$, let

$$
\|u\|_{N^{1, p}(X)}=\left(\int_{X}|u|^{p} d \mu+\inf _{g} \int_{X} g^{p} d \mu\right)^{1 / p},
$$


where the infimum is taken over all $p$-weak upper gradients of $u$. The Newtonian space on $X$ is the quotient space

$$
N^{1, p}(X)=\left\{u:\|u\|_{N^{1, p}(X)}<\infty\right\} / \sim,
$$

where $u \sim v$ if and only if $\|u-v\|_{N^{1, p}(X)}=0$.

Definition 2.4. We say that $X$ supports a weak $(1, p)$-Poincaré inequality if there exist constants $c_{P}>0$ and $\tau \geq 1$ such that for all balls $B$ of $X$, all locally integrable functions $u$ on $X$ and for all $p$-weak upper gradients $g$ of $u$, we have

$$
f_{B}\left|u-u_{B}\right| d \mu \leq c_{P} r\left(f_{\tau B} g^{p} d \mu\right)^{1 / p},
$$

where

$$
u_{B}=f_{B} u d \mu=\frac{1}{\mu(B)} \int_{B} u d \mu
$$

and $r$ is the pre-assigned radius of the ball $B$.

Definition 2.6. Let $1 \leq p<\infty$. The variational $p$-capacity of a set $E \subset X$ is the number

$$
\operatorname{cap}_{p}(E)=\inf \|g\|_{L^{p}(X)}^{p},
$$

where the infimum is taken over all nonnegative Borel measurable functions $g$ which are $p$-weak upper gradients of some function $u \in N^{1, p}(X)$ that satisfies $u=1$ on $E$. If there are no functions which satisfy the requirements, then we set $\operatorname{cap}_{p}(E)=\infty$.

Observe that if $\mu(X)<\infty$, then the constant function one will do as a test function and all sets are of zero capacity.

We point out here that the functions in $N^{1, p}(X)$ are necessarily $p$ quasicontinuous (see $[\mathrm{BBS}]$ ) and thus the above definition of cap $\mathrm{p}_{p}$ agrees with the classical definition of variational capacity where the functions are required in addition to satisfy $u=1$ in a neighbourhood of $E$ (see $[\mathrm{FZ}]$ or $[\mathrm{EG}]$ ). The classical definition is needed if $X=\mathbb{R}^{n}$ and we replace the requirement $u \in N^{1, p}\left(\mathbb{R}^{n}\right)$ with $u \in W^{1, p}\left(\mathbb{R}^{n}\right)$, because functions in $W^{1, p}\left(\mathbb{R}^{n}\right)$ are not necessarily $p$-quasicontinuous and can be arbitrarily perturbed on a set of Lebesgue measure zero. However, for every function in $W^{1, p}\left(\mathbb{R}^{n}\right)$ there is a $p$-quasicontinuous representative and the definitions coincide.

Since the norm of the upper gradient does not increase under truncation, we see that the same number is obtained if we restrict to the functions $0 \leq u \leq 1$ for which $u=1$ on $E$.

Throughout the paper, we assume that the measure $\mu$ is doubling, $\mu(X)=\infty$ and that $X$ is proper, that is, closed and bounded sets are compact. We recall that a metric space with a doubling measure is 
proper if and only if the space is complete. In addition, we assume that $X$ supports a weak $(1,1)$-Poincaré inequality. It follows that the space is quasiconvex and hence uniformly perfect, see for example [Ke].

Under these assumptions, the variational $p$-capacity enjoys the standard properties of capacities, see for example [EG]. In particular, the p-capacity is a Choquet capacity and consequently for all Borel sets $E$ (more generally, for all analytic sets), we have

$$
\operatorname{cap}_{p}(E)=\sup \left\{\operatorname{cap}_{p}(K): K \subset E, K \text { compact }\right\} \text {. }
$$

It is essential for us that these properties hold true for all values $1 \leq$ $p<\infty$. For this we refer to [Ch]. We mainly work with compact sets, but since we have Choquet capacities this is not a serious restriction.

There are several definitions for capacities. The Sobolev p-capacity of a set $E \subset X$ is

$$
\mathrm{C}_{p}(E)=\inf \|u\|_{N^{1, p}(X)}^{p},
$$

where the infimum is taken over all functions $u \in N^{1, p}(X)$ for which $u=1$ on $E$. As with the variational $p$-capacity, we do not require $u=1$ in a neighbourhood of $E$. With our assumptions on $X$, the variational $p$-capacity and the Sobolev $p$-capacity have the same null sets under the assumption that $X$ is $p$-hyperbolic, see the last section. The Sobolev capacity is the correct gauge for distinguishing between two Newtonian functions. We say that a property holds p-quasieverywhere if the set of points for which the property does not hold has Sobolev $p$-capacity zero. If $u \in N^{1, p}(X)$, then $u \sim v$ if and only if $u=v p$-quasieverywhere. Moreover, Corollary 3.3 in Shanmugalingam [Sh] shows that if $u, v \in$ $N^{1, p}(X)$ and $u=v \mu$-a.e., then $u \sim v$.

Next we recall the definition and basic properties of functions of bounded variation on metric spaces, see Miranda [Mi].

Definition 2.7. For $u \in L_{\text {loc }}^{1}(X)$, we define

$$
\begin{aligned}
& \|D u\|(X) \\
& =\inf \left\{\liminf _{i \rightarrow \infty} \int_{X} g_{u_{i}} d \mu: u_{i} \in \operatorname{Lip}_{\mathrm{loc}}(X), u_{i} \rightarrow u \text { in } L_{\mathrm{loc}}^{1}(X)\right\},
\end{aligned}
$$

where $g_{u_{i}}$ is a 1 -weak upper gradient of $u_{i}$. We say that a function $u \in L^{1}(X)$ is of bounded variation, $u \in B V(X)$, if $\|D u\|(X)<\infty$. Moreover, a measurable set $E \subset X$ is said to have finite perimeter if $\left\|D \chi_{E}\right\|(X)<\infty$.

Observe that in [Mi] the functions of bounded variation are defined in terms of the local Lipschitz constant

$$
\operatorname{Lip} u(x)=\liminf _{r \rightarrow 0} \sup _{\substack{y \in B(x, r) \\ 5}} \frac{|u(x)-u(y)|}{d(x, y)},
$$


but we may use the 1-weak upper gradient instead. Note that if $u$ is a locally Lipschitz continuous function, its local Lipschitz constant is an upper gradient of $u$. We observe that all results of [Mi] hold for upper gradients as well. By replacing $X$ with an open set $U \subset X$, we may define $\|D u\|(U)$. We denote

$$
P(E, U)=\left\|D \chi_{E}\right\|(U) .
$$

From Theorem 3.4 in [Mi], we have that $\|D u\|$ is a Borel regular measure (restricted to the open sets of $X$ ) with finite mass.

If the space supports a weak $(1,1)$-Poincaré inequality, then for every $u \in B V(X)$, we have

$$
\int_{B}\left|u-u_{B}\right| d \mu \leq c_{P} r\|D u\|(\tau B),
$$

where the constant $c_{P}$ and the dilation factor $\tau \geq 1$ are the same constants as in (2.5), and $r$ is the pre-assigned radius of $B$. If we set $u=\chi_{E}$, where $E$ is a set of finite perimeter, we get the relative isoperimetric inequality

$$
\min \{\mu(B \cap E), \mu(B \backslash E)\} \leq 2 c_{P} r P(E, \tau B) .
$$

The following coarea formula will be useful for us.

Theorem 2.11 (Coarea formula). If $u \in B V(X)$ and $A \subset X$ is a Borel set, then

$$
\|D u\|(A)=\int_{-\infty}^{\infty} P(\{u>\lambda\}, A) d \lambda .
$$

For a proof, see Proposition 4.2 in [Mi].

\section{Equivalence of the Capacity and the Hausdorff CONTENT}

In this section, we show that the variational 1-capacity is equivalent to a Hausdorff content. Our main tool is the following metric space version of boxing inequality.

Theorem 3.1 (Boxing inequality). Let $U \subset X$ be an open set of finite perimeter with $\mu(U)<\infty$ and $\tau$ the dilation constant in (2.5). Then there exists a collection of disjoint balls $B\left(x_{i}, \tau r_{i}\right), i=1,2, \ldots$, such that

$$
\begin{gathered}
U \subset \bigcup_{i=1}^{\infty} B\left(x_{i}, 5 \tau r_{i}\right), \\
\frac{1}{2 c_{D}}<\frac{\mu\left(U \cap B\left(x_{i}, r_{i}\right)\right)}{\mu\left(B\left(x_{i}, r_{i}\right)\right)} \leq \frac{1}{2}
\end{gathered}
$$


for $i=1,2, \ldots$, and

$$
\sum_{i=1}^{\infty} \frac{\mu\left(B\left(x_{i}, 5 \tau r_{i}\right)\right)}{5 \tau r_{i}} \leq c P(U, X)
$$

The constant $c$ depends only on the doubling constant $c_{D}$ and the constants in the weak $(1,1)$-Poincaré inequality.

Proof. Let $x \in U$. Since $U$ is open,

$$
\frac{\mu(U \cap B(x, r))}{\mu(B(x, r))}=1
$$

for small radii $r$. Therefore, there exists $r_{x}$ such that

$$
\frac{\mu\left(U \cap B\left(x, r_{x}\right)\right)}{\mu\left(B\left(x, r_{x}\right)\right)}=1 .
$$

Recall that $\mu(X)=\infty$. This implies that

$$
\lim _{r \rightarrow \infty} \frac{\mu(U \cap B(x, r))}{\mu(B(x, r))}=0,
$$

since $\mu(U)<\infty$. Hence, there exists $k=k_{x}$ such that

$$
\frac{\mu\left(U \cap B\left(x, 2^{m} r_{x}\right)\right)}{\mu\left(B\left(x, 2^{m} r_{x}\right)\right)}>\frac{1}{2}
$$

for $m=0,1, \ldots, k-1$, and

$$
\frac{\mu\left(U \cap B\left(x, 2^{k} r_{x}\right)\right)}{\mu\left(B\left(x, 2^{k} r_{x}\right)\right)} \leq \frac{1}{2} .
$$

Because the measure $\mu$ is doubling, we obtain

$$
\frac{\mu\left(U \cap B\left(x, 2^{k} r_{x}\right)\right)}{\mu\left(B\left(x, 2^{k} r_{x}\right)\right)} \geq \frac{\mu\left(U \cap B\left(x, 2^{k-1} r_{x}\right)\right)}{c_{D} \mu\left(B\left(x, 2^{k-1} r_{x}\right)\right)}>\frac{1}{2 c_{D}} .
$$

Denote $R_{x}=2^{k} r_{x}$. Since $U$ is measurable, we obtain by (3.2) that

$$
\begin{aligned}
\mu\left(B\left(x, R_{x}\right) \backslash U\right) & =\mu\left(B\left(x, R_{x}\right)\right)-\mu\left(B\left(x, R_{x}\right) \cap U\right) \\
& \geq \mu\left(U \cap B\left(x, R_{x}\right)\right) .
\end{aligned}
$$

This implies that

$$
\begin{aligned}
\min & \left\{\mu\left(B\left(x, R_{x}\right) \cap U\right), \mu\left(B\left(x, R_{x}\right) \backslash U\right)\right\} \\
& =\mu\left(B\left(x, R_{x}\right) \cap U\right) \geq \frac{1}{2 c_{D}} \mu\left(B\left(x, R_{x}\right)\right) .
\end{aligned}
$$

By the relative isoperimetric inequality (2.10) and the previous estimate, we obtain

$$
\frac{\mu\left(B\left(x, R_{x}\right)\right)}{R_{x}} \leq c P\left(U, B\left(x, \tau R_{x}\right)\right)
$$


We apply a covering argument to the family of balls $B\left(x, \tau R_{x}\right), x \in U$, to obtain pairwise disjoint balls $B\left(x_{i}, \tau R_{i}\right), i=1,2, \ldots$, such that

$$
\bigcup_{x \in U} B\left(x, \tau R_{x}\right) \subset \bigcup_{i=1}^{\infty} B\left(x_{i}, 5 \tau R_{i}\right) .
$$

Finally, we conclude that

$$
\begin{aligned}
& \sum_{i=1}^{\infty} \frac{\mu\left(B\left(x_{i}, 5 \tau R_{i}\right)\right)}{5 \tau R_{i}} \leq c \sum_{i=1}^{\infty} \frac{\mu\left(B\left(x_{i}, R_{i}\right)\right)}{R_{i}} \\
& \quad \leq c \sum_{i=1}^{\infty} P\left(U, B\left(x_{i}, \tau R_{i}\right)\right)=c P\left(U, \bigcup_{i=1}^{\infty} B\left(x_{i}, \tau R_{i}\right)\right) \\
& \quad \leq c P(U, X) .
\end{aligned}
$$

Here we also used the facts that the balls are disjoint and that $P(U, \cdot)$ is a Borel measure.

Next we define a Hausdorff content by applying the Carathéodory construction to the function

$$
h(B(x, r))=\frac{\mu(B(x, r))}{r} .
$$

The restricted spherical Hausdorff content of codimension one on $X$ is defined as

$$
\mathcal{H}_{R}^{h}(E)=\inf \left\{\sum_{i=1}^{\infty} h\left(B\left(x_{i}, r_{i}\right)\right): E \subset \bigcup_{i=1}^{\infty} B\left(x_{i}, r_{i}\right), r_{i} \leq R\right\},
$$

where $0<R<\infty$. In the above definition, we allow for the possibility that $r_{i}=0$ for some $i$, with the convention that $B\left(x_{i}, 0\right)=\emptyset$ and $h(\emptyset)=0$.

When $R=\infty$,

$$
\mathcal{H}_{\infty}^{h}(E)=\inf \left\{\sum_{i=1}^{\infty} h\left(B\left(x_{i}, r_{i}\right)\right): E \subset \bigcup_{i=1}^{\infty} B\left(x_{i}, r_{i}\right), r_{i}<\infty\right\}
$$

is the Hausdorff content of E. The Hausdorff measure of codimension one of $E \subset X$ is defined as

$$
\mathcal{H}^{h}(E)=\lim _{R \rightarrow 0} \mathcal{H}_{R}^{h}(E)
$$

Remark 3.3. (1) By examining the proof of the boxing inequality (Theorem 3.1), we see that instead of an open set $U$ the claim holds true for a $\mu$-measurable set $E$ of finite perimeter with $\mu(E)<\infty$. In this case, we can cover all points $x$ for which

$$
\sup _{r>0} \frac{\mu(E \cap B(x, r))}{\mu(B(x, r))} \geq \gamma>0
$$


with balls as in the boxing inequality, but now the lower bound $1 / 2 c_{D}$ is replaced with $\gamma / 2 c_{D}$, and the constant $c$ depends on $\gamma$.

(2) The boxing inequality gives us the following useful upper bound

$$
\mathcal{H}_{\infty}^{h}(E) \leq c \inf \{P(U, X): E \subset U, U \text { is open, } \mu(U)<\infty\} .
$$

(3) Furthermore, if $R>0$ is such that for every $x \in E$ there is $r_{x}>0$ with $2 r_{x}<R$ such that

$$
\frac{\mu\left(E \cap B\left(x, r_{x}\right)\right)}{\mu\left(B\left(x, r_{x}\right)\right)}>\frac{1}{2}
$$

and

$$
\frac{\mu\left(E \cap B\left(x, 2 r_{x}\right)\right)}{\mu\left(B\left(x, 2 r_{x}\right)\right)} \leq \frac{1}{2},
$$

then the cover can be taken with the additional restriction that $r_{i}<R$. Thus we obtain estimates for $\mathcal{H}_{R}^{h}(E)$ rather than $\mathcal{H}_{\infty}^{h}(E)$. In particular, if $E \subset B\left(x_{0}, R\right)$ for some $x_{0} \in X$ and $R>0$, then

$$
\mathcal{H}_{2 R}^{h}(E) \leq c \inf \left\{P(U, X): E \subset U \subset B\left(x_{0}, 4 R\right), U \text { open }\right\} .
$$

Our next goal is to show that the capacity of order one and the Hausdorff content are equivalent.

Lemma 3.4. For any set $E \subset X$, we have

$$
\operatorname{cap}_{1}(E) \leq c_{D} \mathcal{H}_{\infty}^{h}(E)
$$

where $c_{D}$ is the doubling constant of $\mu$.

Proof. If $\mathcal{H}_{\infty}^{h}(E)=\infty$, there is nothing to prove. Therefore, without loss of generality, we may assume that $\mathcal{H}_{\infty}^{h}(E)<\infty$. Let $\varepsilon>0$ and $B\left(x_{i}, r_{i}\right), i \in I \subset \mathbb{N}$, be a covering of $E$ such that

$$
\mathcal{H}_{\infty}^{h}(E)>\sum_{i \in I} \frac{\mu\left(B\left(x_{i}, r_{i}\right)\right)}{r_{i}}-\varepsilon .
$$

By setting

$$
u_{i}(x)=\left(1-\frac{\operatorname{dist}\left(x, B\left(x_{i}, r_{i}\right)\right)}{r_{i}}\right)_{+},
$$

for $i \in I$, and observing that $g_{i}=\chi_{B\left(x_{i}, 2 r_{i}\right)} / r_{i}$ is a weak upper gradient of $u_{i}$, we obtain the following upper bound

$$
\begin{aligned}
\operatorname{cap}_{1}(E) & \leq \sum_{i \in I} \operatorname{cap}_{1}\left(B\left(x_{i}, r_{i}\right)\right) \\
& \leq \sum_{i \in I} \int_{X} g_{i} d \mu=\sum_{i \in I} \frac{\mu\left(B\left(x_{i}, 2 r_{i}\right)\right)}{r_{i}} \\
& \leq c_{D} \sum_{i \in I} \frac{\mu\left(B\left(x_{i}, r_{i}\right)\right)}{r_{i}}<c_{D}\left(\mathcal{H}_{\infty}^{h}(E)+\varepsilon\right) .
\end{aligned}
$$


Letting $\varepsilon \rightarrow 0$, we obtain the desired result.

Remark 3.5. (1) The proof of Lemma 3.4 also applies when $1<p<\infty$. In this case, we obtain

$$
\operatorname{cap}_{p}(E) \leq c_{D} \mathcal{H}_{\infty}^{h}(E)
$$

where

$$
h(B(x, r))=\frac{\mu(B(x, r))}{r^{p}}
$$

and $\mathcal{H}_{\infty}^{h}(E)$ is the spherical Hausdorff content of codimension $p$.

(2) By a modification of the proof of Lemma 3.4, we can see that if $E \subset B\left(x_{0}, R / 2\right)$, then

$$
\mathrm{C}_{1}(E) \leq(1+R) c_{D} \mathcal{H}_{R}^{h}(E) .
$$

The next result gives the equivalence of the 1-capacity and the Hausdorff content of codimension one. We provide an argument based on the boxing inequality.

Theorem 3.6. Let $K$ be a compact subset of $X$. Then

$$
\frac{1}{c} \operatorname{cap}_{1}(K) \leq \mathcal{H}_{\infty}^{h}(K) \leq c \operatorname{cap}_{1}(K),
$$

where $c$ depends only on the doubling constant $c_{D}$ and the constants in the weak $(1,1)$-Poincaré inequality.

Proof. By Lemma 3.4, we have

$$
\operatorname{cap}_{1}(K) \leq c_{D} \mathcal{H}_{\infty}^{h}(K) .
$$

We now prove the other direction. Let $\varepsilon>0$. By a result of [KS], we may choose a compactly supported Lipschitz continuous function $u$ such that $u=1$ in $K, 0 \leq u \leq 1$ and

$$
\int_{X} g d \mu \leq \operatorname{cap}_{1}(K)+\varepsilon
$$

for some 1-weak upper gradient $g$ of $u$.

By the coarea formula (Theorem 2.11), we have

$$
\int_{0}^{1} P(\{u>\lambda\}, X) d \lambda=\|D u\|(X) .
$$

If

$$
P(\{u>\lambda\}, X)>\|D u\|(X)
$$

for every $\lambda$ with $0<\lambda<1$, then we get the contradiction

$$
\|D u\|(X)=\int_{0}^{1} P(\{u>\lambda\}, X) d \lambda>\|D u\|(X) .
$$


Thus for some $\lambda_{0}$ with $0<\lambda_{0}<1$, we have

$$
P\left(\left\{u>\lambda_{0}\right\}, X\right) \leq\|D u\|(X) \leq \int_{X} g d \mu \leq \operatorname{cap}_{1}(K)+\varepsilon .
$$

The set $U=\left\{u>\lambda_{0}\right\}$ is an open neighborhood of $K$ and $\mu(U)<\infty$.

By Remark 3.3(2), we obtain

$$
\mathcal{H}_{\infty}^{h}(K) \leq \mathcal{H}_{\infty}^{h}(U) \leq c P(U, X) \leq c\left(\operatorname{cap}_{1}(K)+\varepsilon\right)
$$

Since $\varepsilon$ is arbitrary, this completes the proof.

\section{LEBESGUE POINTS}

The next result shows that, under our standing assumptions, functions in $N^{1,1}(X)$ have Lebesgue points outside a set of 1-capacity zero, and that they can be obtained by taking the limit of integral averages over small balls. Note that functions in $N^{1,1}(X)$ are always defined 1-quasieverywhere and they are 1-quasicontinuous, see Theorem 1.1 in [BBS].

Theorem 4.1. Suppose that $u \in N^{1,1}(X)$. Then there is $E \subset X$ such that $\operatorname{cap}_{1}(E)=0$ and

$$
\lim _{r \rightarrow 0} \int_{B(x, r)}|u-u(x)| d \mu=0
$$

for every $x \in X \backslash E$.

Remark 4.2. A modification of the proof of Theorem 4.1 given here will yield the stronger result that $\mathrm{C}_{1}(E)=0$. We will include appropriate comments in this section to demonstrate this fact.

The corresponding result for $p>1$ on metric spaces has been studied in [KL]. The proof for $p=1$ is rather straightforward adaptation of the classical proof, see [FZ] and [EG], except for the following capacitary weak type estimate. In $\mathbb{R}^{n}$, this estimate is usually proved by Besicovitch covering theorem, extension results or representation formulas, see [FZ] or Lemma 1 on page 158 of [EG]. We do not have these tools available. The method in [KL] cannot be extended to our case either, because it requires that the Hardy-Littlewood maximal operator is bounded in $L^{p}(X)$, which is not true for $p=1$.

We recall that the Hardy-Littlewood maximal function of a locally integrable function $u$ is defined as

$$
M u(x)=\sup f_{B}|u| d \mu,
$$

where the supremum is taken over all open balls $B$ which contain $x$. 
Lemma 4.3. If $u \in B V(X)$, then

$$
\operatorname{cap}_{1}(\{x \in X: M u(x)>\lambda\}) \leq \frac{c}{\lambda}\|D u\|(X)
$$

for every $\lambda>0$. The constant $c$ depends only on the doubling constant $c_{D}$ and the constants in the weak $(1,1)$-Poincaré inequality.

Proof. Let $u_{\lambda}=u / \lambda$. Clearly $u_{\lambda} \in B V(X)$ and (4.4) holds if and only if

$$
\operatorname{cap}_{1}\left(\left\{x \in X: M u_{\lambda}(x)>1\right\}\right) \leq c\left\|D u_{\lambda}\right\|(X) .
$$

Thus we may assume without loss of generality that $\lambda=1$. We may also assume that $u \geq 0$.

Let

$$
E=\{x \in X: M u(x)>1\} .
$$

We apply a covering argument to find a disjoint family of balls $B\left(x_{i}, \tau r_{i}\right)$, $i=1,2, \ldots$, such that

$$
E \subset \bigcup_{i=1}^{\infty} B\left(x_{i}, 5 \tau r_{i}\right)
$$

and $u_{B\left(x_{i}, r_{i}\right)}>1$ for every $i=1,2, \ldots$ Here $\tau$ is the dilation constant in $(2.5)$.

Define

$$
F_{i}=\left\{x \in B\left(x_{i}, r_{i}\right): u(x) \leq 1 / 2\right\}
$$

and let $I$ be the set of indices $i$ satisfying

$$
\mu\left(F_{i}\right)<\frac{1}{2} \mu\left(B\left(x_{i}, r_{i}\right)\right) .
$$

Let us first consider the balls $B\left(x_{i}, r_{i}\right)$ with $i \notin I$. Then

$$
\mu\left(F_{i}\right) \geq \frac{1}{2} \mu\left(B\left(x_{i}, r_{i}\right)\right)
$$

and it follows that

$$
\int_{B\left(x_{i}, r_{i}\right)}\left|u-u_{B\left(x_{i}, r_{i}\right)}\right| d \mu \geq \int_{F_{i}}\left|u-u_{B\left(x_{i}, r_{i}\right)}\right| d \mu \geq \mu\left(B\left(x_{i}, r_{i}\right)\right) / 4 .
$$

Here we also used the fact that $\left|u-u_{B\left(x_{i}, r_{i}\right)}\right|>1 / 2$ in the set $F_{i}$ since $u_{B\left(x_{i}, r_{i}\right)}>1$.

The doubling condition of $\mu$ and the weak $(1,1)$-Poincaré inequality (2.9) imply that

$$
\begin{aligned}
\mathcal{H}_{\infty}^{h}\left(B\left(x_{i}, 5 \tau r_{i}\right)\right) & \leq \frac{\mu\left(B\left(x_{i}, 5 \tau r_{i}\right)\right)}{5 \tau r_{i}} \leq c \frac{\mu\left(B\left(x_{i}, r_{i}\right)\right)}{r_{i}} \\
& \leq \frac{c}{r_{i}} \int_{B\left(x_{i}, r_{i}\right)}\left|u-u_{B\left(x_{i}, r_{i}\right)}\right| d \mu \\
& \leq c \| \operatorname{di\| }\left(B\left(x_{i}, \tau r_{i}\right)\right) .
\end{aligned}
$$


Next we consider the case $i \in I$, that is

$$
\mu\left(\left\{x \in B\left(x_{i}, r_{i}\right): u(x)>1 / 2\right\}\right)>\frac{1}{2} \mu\left(B\left(x_{i}, r_{i}\right)\right) .
$$

It follows from the doubling of the measure $\mu$ that there exists $\gamma>0$, depending only on $c_{D}$ and $\tau$, such that

$$
\bigcup_{i \in I} B\left(x_{i}, 5 \tau r_{i}\right) \subset\left\{x \in X: \sup _{r>0} \frac{\mu(B(x, r) \cap\{u>\alpha\})}{\mu(B(x, r))} \geq \gamma\right\}
$$

for every $\alpha \in[1 / 4,1 / 2]$. We denote the set on the right hand side of (4.7) by $A_{\alpha}$.

By Theorem 3.1 and Remark 3.3(1), it follows that for every $\alpha \in$ $[1 / 4,1 / 2]$, there exists a covering $B\left(y_{i}, 5 \tau \rho_{i}\right), i=1,2, \ldots$, of $A_{\alpha}$ such that

$$
\mathcal{H}_{\infty}^{h}\left(A_{\alpha}\right) \leq \sum_{i=1}^{\infty} \frac{\mu\left(B\left(y_{i}, 5 \tau \rho_{i}\right)\right)}{5 \tau \rho_{i}} \leq c P(\{u>\alpha\}, X) .
$$

As in the proof of Theorem 3.6, by the coarea formula (Theorem 2.11), there exists $\alpha_{0} \in(1 / 4,1 / 2)$ such that

$$
P\left(\left\{u>\alpha_{0}\right\}, X\right) \leq 4\|D u\|(X) .
$$

Thus

$$
\mathcal{H}_{\infty}^{h}\left(\bigcup_{i \in I} B\left(x_{i}, 5 \tau r_{i}\right)\right) \leq \mathcal{H}_{\infty}^{h}\left(A_{\alpha_{0}}\right) \leq c\|D u\|(X)
$$

Finally, by Lemma 3.4, (4.6) and (4.8), we conclude that

$$
\begin{aligned}
\operatorname{cap}_{1}(E) & \leq c \mathcal{H}_{\infty}^{h}(\{x \in X: M u(x)>1\}) \\
& \leq c \sum_{i \notin I} \mathcal{H}_{\infty}^{h}\left(B\left(x_{i}, 5 \tau r_{i}\right)\right)+c \mathcal{H}_{\infty}^{h}\left(\bigcup_{i \in I} B\left(x_{i}, 5 \tau r_{i}\right)\right) \\
& \leq c \sum_{i \notin I}\|D u\|\left(B\left(x_{i}, \tau r_{i}\right)\right)+c\|D u\|(X) \\
& \leq c\|D u\|(X) .
\end{aligned}
$$

Here we used the facts that the balls $B\left(x_{i}, \tau r_{i}\right)$ are disjoint and that $\|D u\|(\cdot)$ is a Borel measure.

Remark 4.9. By a modification of the proof of Lemma 4.3, we get also a corresponding estimate for the Sobolev capacity. Indeed,

$$
\mathrm{C}_{1}\left(\left\{x \in X: M_{R} u(x)>\lambda\right\}\right) \leq \frac{c_{R}}{\lambda}\|D u\|(X),
$$

where $M_{R} u$ is the restricted version of the maximal function with balls of radii smaller than or equal to $R$. 
The following well known lemma is needed in the proof of Theorem 4.1. For the proof, we refer to the proof of Theorem 3 on page 77 of [EG] or Lemma 4.3 in [KL].

Lemma 4.10. Let $u \in N^{1,1}(X)$ and

$$
\Lambda=\left\{x \in X: \limsup _{r \rightarrow 0} r \frac{\|D u\|(B(x, r))}{\mu(B(x, r))}>0\right\} .
$$

Then $\mathcal{H}_{\infty}^{h}(\Lambda)=\mathcal{H}^{h}(\Lambda)=0$.

Now we are ready to prove Theorem 4.1.

Proof of Theorem 4.1. Let $u \in N^{1,1}(X)$. By Lemmas 4.10 and 3.4, we have $\operatorname{cap}_{1}(\Lambda)=0$. According to the weak Poincaré inequality,

$$
\lim _{r \rightarrow 0} f_{B(x, r)}\left|u-u_{B(x, r)}\right| d \mu=0
$$

for each $x \notin \Lambda$. By Theorem 4.1 in [Sh], continuous functions are dense in $N^{1,1}(X)$, and therefore we can choose functions $u_{i} \in C(X) \cap N^{1,1}(X)$ such that

$$
\left\|D\left(u-u_{i}\right)\right\|(X) \leq 4^{-i}, \quad i=1,2, \ldots
$$

Set

$$
F_{i}=\left\{x \in X: M\left(u-u_{i}\right)>2^{-i}\right\} .
$$

According to Lemma 4.3,

$$
\operatorname{cap}_{1}\left(F_{i}\right) \leq c 2^{i}\left\|D\left(u-u_{i}\right)\right\|(X) \leq c 2^{-i} .
$$

Furthermore, since

$$
\left|u_{B(x, r)}-u_{i}(x)\right| \leq f_{B(x, r)}\left|u-u_{i}\right| d \mu+f_{B(x, r)}\left|u_{i}-u_{i}(x)\right| d \mu
$$

and functions $u_{i}$ are continuous, the definition of $F_{i}$ implies that

$$
\limsup _{r \rightarrow 0}\left|u_{B(x, r)}-u_{i}(x)\right| \leq M\left(u-u_{i}\right)(x) \leq 2^{-i}
$$

for every $x \notin \Lambda \cup F_{i}$. Set

$$
E_{k}=\Lambda \cup\left(\bigcup_{j=k}^{\infty} F_{j}\right)
$$

Then

$$
\operatorname{cap}_{1}\left(E_{k}\right) \leq \operatorname{cap}_{1}(\Lambda)+\sum_{j=k}^{\infty} \operatorname{cap}_{1}\left(F_{j}\right) \leq c \sum_{j=k}^{\infty} 2^{-j}=c 2^{-k}
$$

Furthermore, if $x \in X \backslash E_{k}$, and $i, j>k$, then

$$
\begin{aligned}
\left|u_{i}(x)-u_{j}(x)\right| & \leq \limsup _{r \rightarrow 0}\left|u_{B(x, r)}-u_{i}(x)\right|+\limsup _{r \rightarrow 0}\left|u_{B(x, r)}-u_{j}(x)\right| \\
& \leq 2^{-i}+2^{-j} .
\end{aligned}
$$


Hence, $\left\{u_{i}\right\}_{i}$ converges uniformly on $X \backslash E_{k}$ to a continuous function $v$, and

$$
\limsup _{r \rightarrow 0}\left|v(x)-u_{B(x, r)}\right| \leq\left|v(x)-u_{i}(x)\right|+\limsup _{r \rightarrow 0}\left|u_{i}(x)-u_{B(x, r)}\right|,
$$

so that (4.11) implies

$$
v(x)=\lim _{r \rightarrow 0} f_{B(x, r)} u d \mu
$$

for every $x \in X \backslash E_{k}$. Now set $E=\cap_{k=1}^{\infty} E_{k}$. Then

$$
\operatorname{cap}_{1}(E) \leq \lim _{k \rightarrow \infty} \operatorname{cap}_{1}\left(E_{k}\right)=0
$$

and

$$
v(x)=\lim _{r \rightarrow 0} f_{B(x, r)} u d \mu
$$

for each $x \in X \backslash E$. We observe that

$$
\begin{aligned}
& \lim _{r \rightarrow 0} \int_{B(x, r)}|u-v(x)| d \mu \\
& \leq \lim _{r \rightarrow 0} f_{B(x, r)}\left|u-u_{B(x, r)}\right| d \mu+\lim _{r \rightarrow 0}\left|u_{B(x, r)}-v(x)\right|=0
\end{aligned}
$$

whenever $x \in X \backslash E$.

The function $v$ is 1-quasicontinuous, because its restriction to $X \backslash E_{k}$ is continuous for every $k=1,2, \ldots$. By Corollary 1.3 in [BBS], for every $\varepsilon>0$ we can find an open set $V_{\varepsilon}$ containing $E$ with capacity no more than $\varepsilon$. By Theorem 1.1 in [BBS], $u$ is 1-quasicontinuous. Hence we may also assume that the restrictions of $u$ and $v$ to $X \backslash V_{\varepsilon}$ are continuous.

By Lebesgue's differentation theorem, we have $u=v \mu$-almost everywhere. It follows from the continuity of the functions that, if $x \in X \backslash V_{\varepsilon}$ and $\mu\left(B(x, r) \backslash V_{\varepsilon}\right)>0$ for every $r>0$, then $u(x)=v(x)$. Consequently

$$
\begin{aligned}
\{x & \left.\in X \backslash V_{\varepsilon}: u(x) \neq v(x)\right\} \\
& \subset\left\{x \in X \backslash V_{\varepsilon}: \mu\left(B(x, r) \backslash V_{\varepsilon}\right)=0 \text { for some } r>0\right\} \\
& \subset\left\{x \in X: M \chi_{V_{\varepsilon}}(x)>1 / 2\right\} \\
& \subset\left\{x \in X: M f_{\varepsilon}(x)>1 / 2\right\},
\end{aligned}
$$

where $f_{\varepsilon} \in N^{1,1}(X)$ is a function satisfying $f_{\varepsilon} \geq 1$ in $V_{\varepsilon}$ and

$$
\left\|D f_{\varepsilon}\right\|(X)<2 \varepsilon \text {. }
$$

Note that $\left\{x \in X: M f_{\varepsilon}(x)>1 / 2\right\}$ is open.

Now we can apply Lemma 4.3 to conclude that

$$
\operatorname{cap}_{1}\left(\left\{x \in X: M f_{\varepsilon}(x)>1 / 2\right\}\right) \leq 2 c\left\|D f_{\varepsilon}\right\|(X) \leq 4 c \varepsilon .
$$


Therefore, $u=v$ in $X \backslash\left(\left\{M f_{\varepsilon}>1 / 2\right\} \cup V_{\varepsilon}\right)$ and, as $\varepsilon \rightarrow 0$, it follows that $u=v 1$-quasieverywhere. The claim follows from this.

Remark 4.12. The fact that $v=u$ can also be proved using a result of Kilpeläinen [Ki]. It states that if two quasicontinuous functions equal almost everywhere, they are actually equal quasieverywhere provided the capacity satisfies the following condition: For all open sets $G \subset X$ and all sets $E \subset X$ with $\mu(E)=0$, we have that $\operatorname{cap}(G)=\operatorname{cap}(G \backslash E)$. To see that cap $_{1}$ satisfies this condition, note that, if $v$ is a function used in computing the capacity $\operatorname{cap}_{1}(G \backslash E$ ), (we may assume that $0 \leq v \leq 1)$, then the function $\widetilde{v}$ given by

$$
\widetilde{v}(x)= \begin{cases}1, & x \in E \cap G, \\ v(x), & x \notin E \cap G,\end{cases}
$$

has the properties that $\widetilde{v} \in N^{1,1}(X), \widetilde{v}$ is admissible for $G$, and any upper gradient for $v$ is a 1 -weak upper gradient of $\widetilde{v}$; hence $\operatorname{cap}_{1}(G) \leq$ $\operatorname{cap}_{1}(G \backslash E)$. As $G \backslash E \subset G$, we also have $\operatorname{cap}_{1}(G) \leq \operatorname{cap}_{1}(G \backslash E)$. This implies the claim.

Remark 4.13. By the use of Remark 4.9 instead of Lemma 4.3, Remark 3.5 instead of Lemma 3.4, and the fact that $\mathrm{C}_{1}(\Lambda) \leq \mathcal{H}_{\infty}^{h}(\Lambda)=0$, we can easily modify the the above proof to conclude the stronger statement that $\mathrm{C}_{1}(E)=0$, where $E$ is as in the above proof.

\section{Capacity and Frostman's lemma}

Let $0<R \leq \infty$. We consider the fractional maximal operator

$$
M^{R} \nu(x)=\sup _{0<r<R} r \frac{\nu(B(x, r))}{\mu(B(x, r))}
$$

of a Radon measure $\nu$. We denote $M^{\infty} \nu=M \nu$.

We recall the following version of Frostman's lemma, which applies in metric spaces.

Theorem 5.1 (Frostman's lemma). Let $U \subset X$ be an open set and $R>0$. Then there exists a Radon measure $\nu$ on $U$ such that

$$
M^{R} \nu \leq 1 \text { on } X
$$

and

$$
\mathcal{H}_{10 R}^{h}(U) \leq c \nu(U)
$$

with $c$ depending only on the doubling constant $c_{D}$.

For a proof, see Theorem 6.1 in [Ma1]. More information about Frostman's lemma can be found, for example, in [Mat]. 
Using the above Frostman's lemma, we obtain the following characterization of the Hausdorff content and hence, by Theorem 3.6, of capacity of order one.

Theorem 5.2. Let $U \subset X$ be an open set. Then

$$
\mathcal{H}_{\infty}^{h}(U) \leq c \sup \left\{\nu(U):\|M \nu\|_{L^{\infty}(X)} \leq 1\right\} \leq c \mathcal{H}_{\infty}^{h}(U),
$$

where the supremum is taken over all Radon measures $\nu$. Here $c$ depends only on the doubling constant $c_{D}$.

Proof. Let $U$ be an open subset of $X$. Then by Frostman's lemma, we obtain a Radon measure $\nu$ such that $M \nu \leq 1 \mu$-almost everywhere on $X$ and

$$
\mathcal{H}_{\infty}^{h}(U) \leq c \nu(U)
$$

This implies the first inequality.

To see the second inequality, we take any cover $\left\{B\left(x_{i}, r_{i}\right)\right\}_{i \in \mathbb{N}}$ of $U$. Let $\nu$ be a Radon measure such that $\|M \nu\|_{L^{\infty}(X)} \leq 1$. Since $M \nu \leq 1$ $\mu$-almost everywhere in $X$, we have

$$
\nu(U) \leq \sum_{i=1}^{\infty} \nu\left(B\left(x_{i}, r_{i}\right)\right) \leq \sum_{i=1}^{\infty} \frac{\mu\left(B\left(x_{i}, r_{i}\right)\right)}{r_{i}} .
$$

The claim follows by taking infimum over all such coverings on the right hand side.

\section{CAPACITY AND FUnCTIONS OF BOUNDED VARIATION}

We may employ different classes of test functions in the definition of the variational capacity. It is clear that we have a more sensitive capacity if the test functions are assumed to be, in addition, continuous or locally Lipschitz continuous. We denote the corresponding capacities with $\operatorname{cap}_{\mathrm{C}, p}(E)$ and $\operatorname{cap}_{\mathrm{L}, p}(E)$, respectively. Thus we have

$$
\operatorname{cap}_{p}(E) \leq \operatorname{cap}_{\mathrm{C}, p}(E) \leq \operatorname{cap}_{\mathrm{L}, p}(E) \text {. }
$$

It has been shown by Kallunki and Shanmugalingam in [KS] that if $X$ is a proper quasiconvex metric measure space equipped with a doubling measure and supporting a weak $(1, p)$-Poincaré inequality, then for any compact set $K \subset X$, we have

$$
\operatorname{cap}_{p}(K)=\operatorname{cap}_{\mathrm{C}, p}(K)=\operatorname{cap}_{\mathrm{L}, p}(K) .
$$

They stated the result only for $1<p<\infty$, but the result holds also when $p=1$.

In this section, we give three alternate definitions for the variational capacity of order one. These definitions are based on functions of bounded 
variation and yield quantities which are equivalent by two sided estimates.

Definition 6.1. Let $K$ be a compact subset of $X$. Then

$$
\operatorname{cap}_{\mathrm{CBV}}(K)=\inf \|D u\|(X),
$$

where the infimum is taken over all compactly supported continuous functions $u \in B V(X)$ such that $u \geq 1$ on a neighborhood of $K$.

Since $u$ is assumed to be continuous, we can require $u$ to satisfy $u \geq 1$ just on $K$ itself to obtain an equivalent definition of $\operatorname{cap}_{\mathrm{CBV}}(K)$. It is not difficult to see that we indeed obtain the same quantity. We may also restrict ourselves to admissible functions $u$ for which we have $0 \leq u \leq 1$.

Theorem 6.2. Let $K$ be a compact subset of $X$. Then the quantities $\operatorname{cap}_{1}(K), \operatorname{cap}_{\mathrm{CBV}}(K)$ and

$$
\inf \{P(U, X): K \subset U, U \text { is open, } \mu(U)<\infty\}
$$

are equivalent by two sided estimates. The constants of comparison in the equivalence of these quantities depend only on the doubling constant $c_{D}$ and the constants in the weak $(1,1)$-Poincaré inequality.

Proof. By Lemma 3.4 and the boxing inequality (Remark 3.3(2)), we have

$$
\begin{aligned}
& \operatorname{cap}_{1}(K) \leq c \mathcal{H}_{\infty}^{h}(K) \\
& \quad \leq c \inf \{P(U, X): K \subset U, U \text { is open, } \mu(U)<\infty\} .
\end{aligned}
$$

Moreover, the inequality

$$
\operatorname{cap}_{\mathrm{CBV}}(K) \leq \operatorname{cap}_{1}(K)
$$

is immediate, since by a result of $[\mathrm{KS}]$, we have

$$
\operatorname{cap}_{1}(K)=\inf \|g\|_{L^{1}(X)},
$$

where the infimum is taken over all compactly supported continuous functions $u \in N^{1,1}(X)$ such that $u \geq 1$ in a neighborhood of $K$ and $g$ is a 1-weak upper gradient of $u$. Since $N^{1,1}(X) \subset B V(X)$, we have

$$
\begin{aligned}
& \operatorname{cap}_{\mathrm{CBV}}(K) \leq \operatorname{cap}_{1}(K) \\
& \quad \leq c \inf \{P(U, X): K \subset U, U \text { is open, } \mu(U)<\infty\} .
\end{aligned}
$$

If $\operatorname{cap}_{\mathrm{CBV}}(K)=\infty$, we have the equivalence of the quantities by (6.3). Thus we may assume that $\operatorname{cap}_{\mathrm{CBV}}(K)<\infty$. Let $\varepsilon>0$. Choose a compactly supported continuous function $u \in B V(X)$ with $0 \leq u \leq 1$ such that $u \geq 1$ in a neighborhood of $K$ and

$$
\|D u\|(X) \leq \operatorname{cap}_{18} \mathrm{CBV}(K)+\varepsilon .
$$


As in the proof of Theorem 3.6, we conclude that by the coarea formula, there exists $\lambda_{0}$ with $0<\lambda_{0}<1$ such that

$$
P\left(\left\{u>\lambda_{0}\right\}, X\right) \leq\|D u\|(X) .
$$

Since $u$ is continuous, the set $\left\{u>\lambda_{0}\right\}$ is open, and as $\lambda_{0}<1$, we have

$$
K \subset\{u \geq 1\} \subset\left\{u>\lambda_{0}\right\} .
$$

Moreover, as $u$ has compact support, $\mu\left(\left\{u>\lambda_{0}\right\}\right)<\infty$. This implies that

$$
\begin{aligned}
& \inf \{P(U, X): K \subset U \text { is open, } \mu(U)<\infty\} \leq P\left(\left\{u>\lambda_{0}\right\}, X\right) \\
& \leq\|D u\|(X) \leq \operatorname{cap}_{\mathrm{CBV}}(K)+\varepsilon .
\end{aligned}
$$

Finally letting $\varepsilon \rightarrow 0$, we get

$$
\inf \{P(U, X): K \subset U, U \text { is open, } \mu(U)<\infty\} \leq \operatorname{cap}_{\mathrm{CBV}}(K) .
$$

By (6.3) we see that the claim is true.

In the following definition, we drop the continuity assumption of the admissible functions.

Definition 6.4. Let $K$ be a compact subset of $X$. Then

$$
\operatorname{cap}_{\mathrm{BV}}(K)=\inf \|D u\|(X),
$$

where the infimum is taken over all $u \in B V(X)$ such that $u=1$ on a neighborhood of $K, 0 \leq u \leq 1$ and the support of $u$ is a compact subset of $X$.

Unlike in the definition of $\operatorname{cap}_{\mathrm{CBV}}(K)$ above, we cannot merely assume that $u=1$ only on $K$ itself in the definition of $\operatorname{cap}_{\mathrm{BV}}(K)$ as then all sets of $\mu$-measure zero will have cap Bv $_{\text {zero. }}$

Theorem 6.5. Let $K$ be a compact subset of $X$. Then

$$
\operatorname{cap}_{\mathrm{BV}}(K) \leq \operatorname{cap}_{\mathrm{CBV}}(K) \leq c \operatorname{cap}_{\mathrm{BV}}(K),
$$

where the constant $c$ depends only on the doubling constant $c_{D}$ and the constants in the weak $(1,1)$-Poincaré inequality.

Proof. Clearly

$$
\operatorname{cap}_{\mathrm{BV}}(K) \leq \operatorname{cap}_{\mathrm{CBV}}(K) .
$$

We may assume that $\operatorname{cap}_{\mathrm{BV}}(K)<\infty$. Let $u \in B V(X)$ such that $u=1$ on a neighborhood $U$ of $K, 0 \leq u \leq 1$, the support of $u$ is a compact subset of $X$, and

$$
\|D u\|(X) \leq \operatorname{cap}_{\mathrm{BV}}(K)+\varepsilon
$$

Since $K$ is compact, we have $\operatorname{dist}(K, X \backslash U)=\delta>0$. 
By the doubling property of the measure, for every $k \in \mathbb{N}$, we may cover $X$ by countably many balls $\left\{B_{i, k}\right\}_{i \in I_{k}}, I_{k} \subset \mathbb{N}$ with radii $2^{-(k+2)} \delta$, such that

if $i \neq j$, and

$$
\frac{1}{2} B_{i, k} \cap \frac{1}{2} B_{j, k}=\emptyset
$$

$$
\sup _{x \in X} \sum_{i \in I_{k}} \chi_{4 \tau B_{i, k}}(x) \leq c,
$$

where $\tau \geq 1$ is the scalar in the weak $(1,1)$-Poincaré inequality. Here $c$ is independent of $k$. Condition (6.6) simply means that the balls $\left\{4 \tau B_{i, k}\right\}_{i \in I_{k}}$, are of bounded overlap. Let $\left\{\varphi_{i, k}\right\}_{i \in I_{k}}$ be a partition of unity subordinate to the cover $\left\{B_{i, k}\right\}_{i \in I_{k}}$ with spt $\varphi_{i, k} \subset 2 B_{i, k}$ and $\varphi_{i, k}$ are $c 2^{k} \delta^{-1}$-Lipschitz continuous on $X$. We define the discrete convolution $u_{k}$ by

$$
u_{k}(x)=\sum_{i \in I_{k}} \varphi_{i, k}(x) u_{B_{i, k}} .
$$

As the support of $u$ is compact, so is the support of $u_{k}$. Moreover, the functions $u_{k}$ are locally Lipschitz continuous, since $\varphi_{i, k}$ are Lipschitz continuous. From this we conclude that $u_{k} \in B V(X) \cap C(X)$. Furthermore $0 \leq u_{k} \leq 1$, and since the radius of $B_{i, k}$ is at most $\delta / 4, u_{k}=1$ on an open neighborhood of $K$.

Fix $x, y \in B_{j, k}$. By the properties of the partition of unity, we obtain

$$
\begin{aligned}
u_{k}(x)-u_{k}(y) & =\sum_{i \in I_{k}}\left(\varphi_{i, k}(x)-\varphi_{i, k}(y)\right) u_{B_{i, k}} \\
& =\sum_{i \in I_{k}}\left(\varphi_{i, k}(x)-\varphi_{i, k}(y)\right)\left(u_{B_{i, k}}-u_{B_{j, k}}\right) .
\end{aligned}
$$

Let

$$
I_{k}^{j}=\left\{i \in I_{k}: 2 B_{i, k} \cap B_{j, k} \neq \emptyset\right\} .
$$

By (6.7), we have

$$
\left|u_{k}(x)-u_{k}(y)\right| \leq c \delta^{-1} 2^{k} d(x, y) \sum_{i \in I_{k}^{j}}\left|u_{B_{i, k}}-u_{B_{j, k}}\right| .
$$

Since $B_{j, k} \subset 4 B_{i, k}$ when $i \in I_{k}^{j}$, we see that by the doubling property of the measure

$$
\begin{aligned}
\left|u_{B_{i, k}}-u_{B_{j, k}}\right| & \leq\left|u_{B_{i, k}}-u_{4 B_{i, k}}\right|+\left|u_{4 B_{i, k}}-u_{B_{j, k}}\right| \\
& \leq c f_{4 B_{i, k}}\left|u-u_{4 B_{i, k}}\right| d \mu+c f_{4 B_{i, k}}\left|u-u_{4 B_{i, k}}\right| d \mu \\
& \leq c f_{4 B_{i, k}}\left|u-u_{4 B_{i, k}}\right| d \mu .
\end{aligned}
$$


Let us denote $\nu=\|D u\|$. By the weak $(1,1)$-Poincaré inequality,

$$
\left|u_{B_{i, k}}-u_{B_{j, k}}\right| \leq c \frac{\delta}{2^{k}} \frac{\nu\left(4 \tau B_{i, k}\right)}{\mu\left(4 B_{i, k}\right)},
$$

from which it follows that

$$
\left|u_{k}(x)-u_{k}(y)\right| \leq c d(x, y) \sum_{i \in I_{k}} \frac{\nu\left(4 \tau B_{i, k}\right)}{\mu\left(B_{i, k}\right)},
$$

where the sum is taken only over those indices for which $x \in 2 B_{i, k}$ or $y \in 2 B_{i, k}$. From (2.8) it follows that the function

$$
g_{k}(x)=c \sum_{i \in I_{k}, x \in 2 B_{i, k}} \frac{\nu\left(4 \tau B_{i, k}\right)}{\mu\left(B_{i, k}\right)},
$$

is an upper gradient of $u_{k}$. Thus

$$
\begin{aligned}
\left\|D u_{k}\right\|(X) & \leq c \int_{X} \sum_{i \in I_{k}, x \in 2 B_{i, k}} \frac{\nu\left(4 \tau B_{i, k}\right)}{\mu\left(B_{i, k}\right)} d \mu(x) \\
& \leq c \sum_{j \in I_{k}} \int_{B_{j, k}} \sum_{i \in I_{k}^{j}} \frac{\nu\left(4 \tau B_{i, k}\right)}{\mu\left(B_{i, k}\right)} d \mu \\
& \leq c \sum_{j \in I_{k}} \sum_{i \in I_{k}^{j}} \int_{B_{j, k}} \frac{\nu\left(4 \tau B_{i, k}\right)}{\mu\left(B_{j, k}\right)} d \mu \\
& \leq c \sum_{j \in I_{k}} \sum_{i \in I_{k}^{j}} \nu\left(4 \tau B_{i, k}\right) .
\end{aligned}
$$

Here the third inequality follows from the doubling condition and the fact that $2 B_{i, k} \cap 2 B_{j, k} \neq \emptyset$ implies that $B_{i, k} \subset 4 B_{j, k}$ and $B_{j, k} \subset 4 B_{i, k}$. By (6.6) and the fact that $\nu=\|D u\|$ is a measure we have

$$
\left\|D u_{k}\right\|(X) \leq c \sum_{i \in I_{k}} \nu\left(4 \tau B_{i, k}\right) \leq c \nu(X) \leq c\left(\operatorname{cap}_{\mathrm{BV}}(K)+\varepsilon\right) .
$$

This implies that

$$
\operatorname{cap}_{\mathrm{CBV}}(K) \leq\left\|D u_{k}\right\|(X) \leq c\left(\operatorname{cap}_{\mathrm{BV}}(K)+\varepsilon\right) .
$$

Letting $\varepsilon \rightarrow 0$, we obtain the claim.

\section{Non-TRIVIALITY OF THE CAPACITY}

Let $U$ be an open and bounded subset of $X$ and $K$ a compact subset of $U$. The relative capacity $\operatorname{cap}_{p}(K, U), 1 \leq p<\infty$, is defined as

$$
\operatorname{cap}_{p}(K, U)=\inf \|g\|_{L^{p}(X)}^{p}
$$

where the infimum is taken over all $u \in N^{1, p}(X)$ such that $u=1$ in $K$, $u=0$ in $X \backslash U$ and $g$ is a $p$-weak upper gradient of $u$. In the same way 
as in the previous section, we can show that $\operatorname{cap}_{1}(K, U), \operatorname{cap}_{\mathrm{CBV}}(K, U)$, $\operatorname{cap}_{\mathrm{BV}}(K, U)$,

$$
\inf \left\{P\left(U^{\prime}, X\right): K \subset U^{\prime}, U^{\prime} \text { is open, } U^{\prime} \Subset U\right\}
$$

and $\mathcal{H}_{2 \operatorname{diam}(U)}^{h}(K)$ are equivalent by two sided estimates. Here the definitions of different relative capacities are obvious modifications of the corresponding global capacities.

Following [Ho], we say that a metric space is p-hyperbolic if there exists a compact set $K \subset X$ so that the relative capacity $\operatorname{cap}_{p}(K, X)$ is strictly positive. Here

$$
\operatorname{cap}_{p}(K, X)=\lim _{i \rightarrow \infty} \operatorname{cap}_{p}\left(C, U_{i}\right),
$$

where $U_{1} \subset U_{2} \subset \ldots$ is an increasing sequence of bounded open subsets of $X$ such that

$$
X=\bigcup_{i=1}^{\infty} U_{i}
$$

If the space is not $p$-hyperbolic, it is said to be $p$-parabolic. Observe that

$$
\operatorname{cap}_{p}(K, X)=\operatorname{cap}_{p}(K) .
$$

Note that $\mathbb{R}^{n}$ endowed with the Lebesgue measure and the Euclidean metric is $p$-parabolic when $p \geq n$ and $p$-hyperbolic if $1 \leq p<n$. More generally, if the measure $\mu$ on a metric space $X$ is $Q$-Ahlfors regular for some $Q \geq 1$, that is

$$
\frac{1}{c} r^{Q} \leq \mu(B(x, r)) \leq c r^{Q}
$$

for some $c \geq 1$ which is independent of $x \in X$ and $r, 0<r<\operatorname{diam}(X)$, then $X$ is $p$-parabolic when $p>Q$ and $p$-hyperbolic when $1 \leq p<Q$. We show that this phenomenon is based on a general principle.

In this section, suppose merely that $\mu$ is doubling and that $X$ supports a weak $(1,1)$-Poincaré inequality. Then $X$ is connected and hence uniformly perfect. From this we conclude that there are $Q_{1}, Q_{2}$ with $0<Q_{1} \leq Q_{2}<\infty$ such that for all $x \in X, 0<r<R<\infty$, and all $y \in B(x, R)$ we have

$$
\frac{1}{c}\left(\frac{r}{R}\right)^{Q_{2}} \leq \frac{\mu(B(y, r))}{\mu(B(x, R))} \leq c\left(\frac{r}{R}\right)^{Q_{1}},
$$

where $c$ depends only on the doubling constant $c_{D}$. The lower bound follows from the doubling property and the upper bound from the weak Poincaré inequality.

The following two results apply even to metric spaces that do not support a weak Poincaré inequality. 
Theorem 7.2. Suppose that the lower bound in (7.1) holds with $Q_{2}>$ 0 . If $p>Q_{2}$, then $X$ is p-parabolic.

Proof. Fix $x_{0} \in X$ and $0<r<R$. Then by the lower bound in (7.1), we have

$$
\frac{\mu\left(B\left(x_{0}, R\right)\right)}{R^{Q_{2}}} \leq c \frac{\mu\left(B\left(x_{0}, r\right)\right)}{r^{Q_{2}}} .
$$

If $p>Q_{2}$ and $R>2 r$, then using the similar test function as in the proof of Lemma 3.4 and the lower bound in (7.1), we have

$$
\begin{aligned}
\operatorname{cap}_{p}\left(\bar{B}\left(x_{0}, r\right), B\left(x_{0}, R\right)\right) & \leq \frac{\mu\left(B\left(x_{0}, R\right)\right)}{(R-r)^{p}} \leq \frac{2^{p}}{R^{p}} \mu\left(B\left(x_{0}, R\right)\right) \\
& \leq \frac{c 2^{p}}{R^{p-Q_{2}}} \frac{\mu\left(B\left(x_{0}, r\right)\right)}{r^{Q_{2}}} \rightarrow 0
\end{aligned}
$$

as $R \rightarrow \infty$. From this we conclude that $\operatorname{cap}_{p}\left(\bar{B}\left(x_{0}, r\right), X\right)=0$ for every $r>0$, and thus $X$ is $p$-parabolic.

In particular, Theorem 7.2 implies that if the measure satisfies (7.1) with $Q_{2}<1$, then $X$ is 1-parabolic. Observe that when $Q_{2}<1$, the space does not support a weak Poincaré inequality.

The following lemma is a metric space version of Theorem 3.5.6 in [Tu].

Lemma 7.3. Let $x_{0} \in X$ and $r>0$. Then

$$
\frac{1}{c} \inf _{r \leq R<\infty} \frac{\mu\left(B\left(x_{0}, R\right)\right)}{R} \leq \mathcal{H}_{\infty}^{h}\left(\bar{B}\left(x_{0}, r\right)\right) \leq \inf _{r \leq R<\infty} \frac{\mu\left(B\left(x_{0}, R\right)\right)}{R},
$$

where $c$ depends only on the doubling constant $c_{D}$.

Proof. The claimed upper bound is clear. We prove the lower bound. By definition

$$
\mathcal{H}_{\infty}^{h}\left(\bar{B}\left(x_{0}, r\right)\right)=\inf \sum_{i \in I} \frac{\mu\left(B_{i}\right)}{r_{i}},
$$

where the infimum is taken over all coverings $\left\{B_{i}\right\}_{i \in I}$ of $\bar{B}\left(x_{0}, r\right)$, where $B_{i}=B\left(x_{i}, r_{i}\right)$. Since the balls in $X$ may have more than one center and radius, it is understood that the cover $\left\{B_{i}\right\}_{i \in I}$ is chosen with preset radii in the above definition. Thus it is possible that the same cover could be used with a different choice of radius to obtain a different number.

Let $\left\{B_{i}\right\}_{i \in I}$ be a cover of $\bar{B}\left(x_{0}, r\right)$. Without loss of generality, we may assume that $B_{i} \cap \bar{B}\left(x_{0}, r\right) \neq \emptyset$ for every $i \in I$. Now there are two possibilities. Assume first that there exists $i_{0}$ for which $r_{i_{0}} \geq r$. Let 
$R_{0}=r_{i_{0}}$. Then it follows that $x_{0} \in 2 B_{i_{0}}$. It follows that $B\left(x_{0}, R_{0}\right) \subset$ $3 B_{i_{0}}$, and by the doubling property, we have

$$
\sum_{i \in I} \frac{\mu\left(B_{i}\right)}{r_{i}} \geq \frac{\mu\left(B_{i_{0}}\right)}{r_{i_{0}}} \geq \frac{1}{c} \frac{\mu\left(3 B_{i_{0}}\right)}{R_{0}} \geq \frac{1}{c} \frac{\mu\left(B\left(x_{0}, R_{0}\right)\right)}{R_{0}} .
$$

As $R_{0} \geq r$, we get

$$
\sum_{i \in I} \frac{\mu\left(B_{i}\right)}{r_{i}} \geq \frac{1}{c} \inf _{r \leq R<\infty} \frac{\mu\left(B\left(x_{0}, R\right)\right)}{R} .
$$

The other possibility is that $r_{i}<r$ for every $i \in I$, which implies that

$$
\sum_{i \in I} \frac{\mu\left(B_{i}\right)}{r_{i}} \geq \frac{1}{r} \sum_{i \in I} \mu\left(B_{i}\right) .
$$

Since $\left\{B_{i}\right\}_{i \in I}$ is a cover of $B\left(x_{0}, r\right)$, we have

$$
\sum_{i \in I} \frac{\mu\left(B_{i}\right)}{r_{i}} \geq \frac{\mu\left(B\left(x_{0}, r\right)\right)}{r} \geq \inf _{r \leq R<\infty} \frac{\mu\left(B\left(x_{0}, R\right)\right)}{R} .
$$

By (7.4) and (7.5), we have the desired claim.

Theorem 7.6. Let $Q_{1}$ be the power in the upper bound in (7.1). If $Q_{1} \geq 1$, then $X$ is 1-hyperbolic.

Proof. By Theorem 3.6, Lemma 7.3 and the upper bound in (7.1), we have

$$
\begin{aligned}
\operatorname{cap}_{1}\left(\bar{B}\left(x_{0}, r\right)\right) & \geq \frac{1}{c} \mathcal{H}_{\infty}^{h}\left(\bar{B}\left(x_{0}, r\right)\right) \geq \frac{1}{c} \inf _{r \leq R<\infty} \frac{\mu\left(B\left(x_{0}, R\right)\right)}{R} \\
& =\frac{1}{c} \inf _{r \leq R<\infty} R^{Q_{1}-1} \frac{\mu\left(B\left(x_{0}, R\right)\right)}{R^{Q_{1}}} \\
& \geq \frac{1}{c} \inf _{r \leq R<\infty} R^{Q_{1}-1} \frac{\mu\left(B\left(x_{0}, r\right)\right)}{r^{Q_{1}}} .
\end{aligned}
$$

If $Q_{1} \geq 1$ we have that

$$
\operatorname{cap}_{1}\left(\bar{B}\left(x_{0}, r\right), X\right)=\operatorname{cap}_{1}\left(\bar{B}\left(x_{0}, r\right)\right) \geq \frac{1}{c} \frac{\mu\left(B\left(x_{0}, r\right)\right)}{r}>0,
$$

and hence $X$ is 1-hyperbolic.

Remark 7.7. It follows from Lemma 7.3 and Theorem 3.6 that $X$ is 1-hyperbolic if and only if

$$
\liminf _{R \rightarrow \infty} \frac{\mu\left(B\left(x_{0}, R\right)\right)}{R}>0
$$

for some (and hence all) $x_{0} \in X$. Thus $X$ is 1-hyperbolic if and only if for all $x_{0} \in X$ the volume $\mu\left(B\left(x_{0}, R\right)\right)$ grows at least as fast as $R$. 
If the measure is $Q$-Ahlfors regular, then the Hausdorff content and the Hausdorff measure have the same null sets. However, as the following example shows, this is not true for a general doubling measure.

Example 7.8. The assumptions that $\mu$ is doubling and that $X$ supports a $(1,1)$-Poincaré inequality do not imply that $X$ is 1-hyperbolic. Let $X=\mathbb{R}^{n}$ equipped with the Euclidean metric and the measure $\mu$ given by

$$
\mu(E)=\int_{E}|x|^{\delta} d x .
$$

Since $w(x)=|x|^{\delta}$ is a Muckenhoupt $A_{1}$-weight when $0<n+\delta<1$, the measure $\mu$ is doubling and supports a $(1,1)$-Poincaré inequality. However, if $n+\delta<1$, the space $X$ is 1 -parabolic, since

$$
\mu(B(0, R))=c \int_{0}^{R} r^{\delta+n-1} d r=c R^{n+\delta},
$$

where $c$ depends only on $n$ and $\delta$, and hence

$$
\frac{\mu(B(0, R))}{R} \leq c R^{n+\delta-1} \rightarrow 0
$$

as $R \rightarrow \infty$. By Lemma 7.3 we have

$$
\mathcal{H}_{\infty}^{h}(B(0, R)) \leq c \inf _{R \leq \rho<\infty} \frac{\mu(B(0, \rho))}{\rho}=0 .
$$

By Lemma 3.4 this implies that $\operatorname{cap}_{1}(\bar{B}(0, R))=0$ for every $R>0$.

Lemma 7.9. Suppose that $X$ is 1-hyperbolic. Then $\mathcal{H}_{\infty}^{h}(E)=0$ if and only if $\mathcal{H}^{h}(E)=0$.

Proof. Let $E \subset X$. As $\mathcal{H}_{\infty}^{h}(E) \leq \mathcal{H}^{h}(E)$, it is easy to see that $\mathcal{H}_{\infty}^{h}(E)=0$ whenever $\mathcal{H}^{h}(E)=0$.

Now suppose that $\mathcal{H}_{\infty}^{h}(E)=0$. In particular, our assumptions and Theorem 7.2 imply that $(7.1)$ holds with $Q_{2} \geq 1$. Without loss of generality, we may assume that $\operatorname{diam}(E)<\infty$. Since $X$ is 1-hyperbolic, there is a compact set $K \subset X$ such that $\operatorname{cap}_{1}(K)>0$. We choose $x_{0} \in E$ and $R>0$ such that $E \subset B\left(x_{0}, R\right)$ and that $K \subset B\left(x_{0}, R\right)$. It follows from Lemma 3.4 that

$$
\mathcal{H}_{\infty}^{h}\left(\bar{B}\left(x_{0}, R\right)\right) \geq \frac{1}{c_{D}} \operatorname{cap}_{1}\left(\bar{B}\left(x_{0}, R\right)\right) \geq \frac{1}{c_{D}} \operatorname{cap}_{1}(K)>0 .
$$

Let $0<\varepsilon<\operatorname{cap}_{1}(K) / c_{D}^{3}$. Since $\mathcal{H}_{\infty}^{h}(E)=0$, we have a cover $\left\{B_{i}\right\}_{i \in I}$, $I \subset \mathbb{N}$, of $E$ such that

$$
\sum_{i \in I} \frac{\mu\left(B_{i}\right)}{r_{i}} \leq \varepsilon
$$


and consequently

$$
\frac{\mu\left(B_{i}\right)}{r_{i}} \leq \varepsilon
$$

for every $i \in I$. Again, without loss of generality, we may assume that $B_{i} \cap E \neq \emptyset$ for every $i \in I$.

If there is an index $i_{0} \in I$ such that $r_{i_{0}} \geq R$, then as $B_{i_{0}} \cap E \neq \emptyset$ and $E \subset \bar{B}\left(x_{0}, R\right)$, we have $\bar{B}\left(x_{0}, r_{i_{0}}\right) \subset 4 \bar{B}_{i_{0}}$. Thus by (7.11) and the doubling property of $\mu$, we have

$$
\begin{aligned}
\varepsilon & \geq \frac{\mu\left(B_{i_{0}}\right)}{r_{i_{0}}} \geq \frac{1}{c_{D}^{2}} \frac{\mu\left(4 B_{i_{0}}\right)}{r_{i_{0}}} \geq \frac{1}{c_{D}^{2}} \frac{\mu\left(\bar{B}\left(x_{0}, r_{i_{0}}\right)\right)}{r_{i_{0}}} \\
& \geq \frac{1}{c_{D}^{2}} \mathcal{H}_{\infty}^{h}\left(\bar{B}\left(x_{0}, R\right)\right) \geq \frac{1}{c_{D}^{3}} \operatorname{cap}_{1}(K) .
\end{aligned}
$$

This is a contradiction, and hence $r_{i}<R$ for every $i \in I$.

Since $B_{i} \cap E \neq \emptyset$ and $r_{i} \leq R$ for every $i \in I$, we have $B_{i} \subset B\left(x_{0}, 4 R\right)$. By (7.1) we get

$$
\frac{\mu\left(B_{i}\right)}{r_{i}} \geq \frac{1}{c} \frac{\mu\left(B\left(x_{0}, 4 R\right)\right)}{(4 R)^{Q_{2}}} r_{i}^{Q_{2}-1}
$$

for every $i \in I$. If $Q_{2}=1,(7.12)$ gives for $\mu\left(B_{i}\right) / r_{i}$ a lower bound that is independent of $r_{i}$. Without loss of generality, we may assume that

$$
\varepsilon<\frac{1}{c} \frac{\mu\left(B\left(x_{0}, 4 R\right)\right)}{(4 R)^{Q_{2}}} .
$$

Hence by (7.11) and (7.12), we have $I=\emptyset$.

This means that $E=\emptyset$ and $\mathcal{H}^{h}(E)=0$.

If $Q_{2}>1$, it follows from (7.12) and (7.11) that

$$
r_{i} \leq\left(\frac{\varepsilon c(4 R)^{Q_{2}}}{\mu\left(B\left(x_{0}, 4 R\right)\right)}\right)^{\frac{1}{Q_{2}-1}}=\delta_{\varepsilon}
$$

for every $i \in I$. Hence

$$
\mathcal{H}_{\delta_{\varepsilon}}^{h}(E) \leq \sum_{i \in I} \frac{\mu\left(B_{i}\right)}{r_{i}} \leq \varepsilon .
$$

Because $Q_{2}>1, \delta_{\varepsilon} \rightarrow 0$ as $\varepsilon \rightarrow 0$. Hence

$$
\mathcal{H}^{h}(E)=\lim _{\varepsilon \rightarrow 0} \mathcal{H}_{\delta_{\varepsilon}}^{h}(E)=0 .
$$

This completes the proof. 
Theorem 7.13. Suppose that $X$ is 1-hyperbolic. Then for all $E \subset X$ we have $\operatorname{cap}_{1}(E)>0$ whenever $\mathrm{C}_{1}(E)>0$.

Proof. It is sufficient to show that if $E \subset X$ is bounded and $\operatorname{cap}_{1}(E)=$ 0 , then $\mathrm{C}_{1}(E)=0$. As $E$ is bounded, there exists $x_{0} \in X$ and $R<\infty$ such that $E \subset B\left(x_{0}, R\right)$.

Because $X$ is 1-hyperbolic, there exists $c>0$ such that

$$
\liminf _{R \rightarrow \infty} \frac{\mu\left(B\left(x_{0}, R\right)\right)}{R} \geq c>0 .
$$

It follows from the doubling of the measure $\mu$ that

$$
\frac{\mu(B(x, r))}{r} \geq \frac{c}{c_{D}}
$$

for every $x \in X$ and $r>R_{0}$ such that $B(x, r) \cap B\left(x_{0}, R\right) \neq \emptyset$. Here $R_{0}>R$ may depend on the ball $B\left(x_{0}, R\right)$.

Let $0<\varepsilon<c / c_{D}$. It follows from Theorem 3.6 that $\mathcal{H}_{\infty}^{h}(E)=0$ and hence there exists a covering $\left\{B\left(x_{i}, r_{i}\right)\right\}_{i}$ such that

$$
\sum_{i=1}^{\infty} \frac{\mu\left(B\left(x_{i}, r_{i}\right)\right)}{r_{i}}<\varepsilon .
$$

If follows from (7.14) that $r_{i}<R_{0}$ for every $i \in \mathbb{N}$. By Remark 3.5(2),

$$
\mathrm{C}_{1}(E) \leq\left(1+R_{0}\right) c_{D} \mathcal{H}_{R_{0}}^{h}(E)<\left(1+R_{0}\right) c_{D} \varepsilon
$$

Letting $\varepsilon \rightarrow 0$, the claim follows.

\section{REFERENCES}

[Ad] Adams, D.R., A note on Choquet integrals with respect to Hausdorff capacity. Function spaces and applications (Lund, 1986), 115-124, Lecture Notes in Math., 1302, Springer, Berlin, 1988.

[BBS] Björn, A., Björn, J., Shanmugalingam, N., Quasicontinuity of NewtonSobolev functions and density of Lipschitz functions on metric spaces. Houston Math. J. (to appear).

[Ch] Choquet, G., Forme abstraite du théorème de capacitabilité, Ann. Inst. Fourier (Grenoble) 9 (1959), 83-89.

[EG] Evans, L.C. and Gariepy, R.F., Measure theory and fine properties of functions, Studies in Advanced Mathematics. CRC Press, Boca Raton, FL, 1992 .

[FZ] Federer, H. and Ziemer, W.P., The Lebesgue set of a function whose distribution derivatives are $p$-th power summable, Indiana Univ. Math. J. 22 (1972/73), 139-158.

[Fl] Fleming, W.H., Functions whose partial derivatives are measures, Illinois J. Math. $41960452-478$.

[Fr] Frostman, O., Potentiel d'équilibre et capacité des ensembles avec quelques applications à la théorie des fonctions, Medd. Lunds Univ. Mat. Sem. 3 (1935), 1-118. 
[Gu] Gustin, W., Boxing inequalities, J. Math. Mech. 9 (1960) 229-239.

[Ho] Holopainen, I., Nonlinear potential theory and quasiregular mappings on Riemannian manifolds, Ann. Acad. Sci. Fenn. Ser. A I Math. Dissertationes 74 (1990).

[KS] Kallunki, S. and Shanmugalingam, N., Modulus and continuous capacity, Ann. Acad. Sci. Fenn. Math. 26 (2001), no. 2, 455-464.

[Ke] Keith, S., Modulus and the Poincaré inequality on metric measure spaces, Math. Z. 245 (2003), no. 2, 255-292.

[Ki] Kilpeläinen, T., A remark on the uniqueness of quasi continuous functions, Ann. Acad. Sci. Fenn. Math. 23 (1998), no. 1, 261-262.

[KL] Kinnunen, J. and Latvala, V., Lebesgue points for Sobolev functions on metric spaces, Rev. Mat. Iberoamericana 18 (2002), 685-700.

[Ma1] Malý, J., Coarea integration in metric spaces, Nonlinear Analysis, Function Spaces and Applications Vol. 7. Proceedings of the Spring School held in Prague July 17-22, 2002. Eds. B. Opic and J. Rakosnik. Math. Inst. of the Academy of Sciences of the Czech Republic, Praha 2003, 142-192.

[Ma2] Malý, J., Coarea properties of Sobolev functions, Function spaces, differential operators and nonlinear analysis (Teistungen, 2001), 371-381, Birkhäuser, Basel, 2003.

[MSZ] Malý, J., Swanson, D., Ziemer, W.P., The co-area formula for Sobolev mappings, Trans. Amer. Math. Soc. 355 (2003), no. 2, 477-492.

[Mat] Mattila, P., Geometry of sets and measures in Euclidean spaces. Fractals and rectifiability. Cambridge Studies in Advanced Mathematics 44, Cambridge University Press, Cambridge, 1995.

[Maz] Maz'ja, V.G, Sobolev spaces. Translated from the Russian by T. O. Shaposhnikova. Springer Series in Soviet Mathematics. Springer-Verlag, Berlin, 1985.

[Mi] Miranda, M., Jr., Functions of bounded variation on "good" metric spaces, J. Math. Pures Appl. (9) 83 (2003), 975-1004.

[Sh] Shanmugalingam, N., Newtonian spaces: An extension of Sobolev spaces to metric measure spaces, Rev. Mat. Iberoamericana 16 (2000), no. 2, $243-279$.

[Sj] Sjödin, T., A note on capacity and Hausdorff measure in homogeneous spaces, Potential Anal. 6 (1997), no. 1, 87-97.

[Tu] Turesson, B.O., Nonlinear potential theory and weighted Sobolev spaces, Lecture Notes in Mathematics 1736, Springer-Verlag, Berlin, 2000.

J.K., Department of Mathematical Sciences, P.O. Box 3000, Fi90014 University of OUlu, Finland

juha.kinnunen@oulu.fi

R.K., Institute of Mathematics, P.O. Box 1100, FI-02015 Helsinki University of TeChNOlogy, Finland

rkorte@math.hut.fi

N.S., Department of Mathematical Sciences, P.O. Box 210025, University of Cincinnati, Cincinnati, OH 45221-0025 U.S.A.

nages@math.uc.edu 
H.T., Department of Mathematics and Statistics, P.O. Box 35 (MAD), FI-40014 University of JyvÄskylä, Finland

tuheli@maths.jyu.fi 